\title{
Neuro-Fuzzy Approach to Forecast Wind Power in Portugal
}

\author{
H.M.I. Pousinho' ${ }^{1}$, V.M.F. Mendes ${ }^{2}$ and J.P.S. Catalão ${ }^{1}$ \\ ${ }^{1}$ Department of Electromechanical Engineering \\ University of Beira Interior \\ R. Fonte do Lameiro, 6200-001 Covilhã (Portugal) \\ Phone: +351 275 329914, Fax: +351 275 329972, e-mail: hmi-21@hotmail.com, catalao@ubi.pt \\ ${ }^{2}$ Department of Electrical Engineering and Automation \\ Instituto Superior de Engenharia de Lisboa \\ R. Conselheiro Emídio Navarro, 1950-062 Lisbon (Portugal) \\ Phone: +351218 317 000, Fax: +351218317 001, e-mail: vfmendes@isel.pt
}

\begin{abstract}
The increased integration of wind power into the electric grid, as nowadays occurs in Portugal, poses new challenges due to its intermittency and volatility. Hence, good forecasting tools play a key role in tackling these challenges. In this paper, a neuro-fuzzy approach is proposed for short-term wind power forecasting in Portugal. Results from a real-world case study are presented. A thorough comparison is carried out, taking into account the results obtained with other approaches. Conclusions are duly drawn.
\end{abstract}

\section{Key words}

Wind power, forecasting, neural networks, fuzzy logic.

\section{Introduction}

Wind generation is the fastest growing source of renewable energy [1]. Indeed, wind-driven power resources have become increasingly important in the planning and operation of electric power systems.

In Portugal, the wind power goal foreseen for 2010 was established by the government as $3750 \mathrm{MW}$, representing about $25 \%$ of the total installed capacity in 2010 [2]. This value has been raised to $5100 \mathrm{MW}$ by the most recent governmental goals for the wind sector.

Hence, Portugal has one of the most ambitious goals in terms of wind power and in 2006 was the second country in Europe with the highest wind power growth.

Short-term wind power forecasting is an extremely important field of research for the energy sector, as the system operators must handle an important amount of fluctuating power from the increasing installed wind power capacity. The time scales concerning short-term prediction are in the order of some days (for the forecast horizon) and from minutes to hours (for the time-step) [3].
In the technical literature, several methods to predict wind power have been reported, namely physical [4] and statistical methods.

Conventional statistical models are identical to the direct random time-series model, including auto regressive (AR), and auto regressive integrated moving average (ARIMA) [5] models. The persistence approach has proven to be a useful first approximation for short-term wind power forecasting.

In the recent years, some new methods are catching researcher's attention, namely neural networks (NN) [6], fuzzy logic [7], evolutionary algorithms [8], and some hybrid methods. The accurate comparison of all the methods is quite difficult because these methods depend on different situations and the data collection is a formidable task.

A hybrid of NN and fuzzy logic, known as adaptivenetwork-based fuzzy inference system (ANFIS), is proposed for short-term wind power forecasting in Portugal.

The proposed approach is compared with persistence, ARIMA and NN approaches, to demonstrate its effectiveness regarding forecasting accuracy and computation time.

\section{Proposed Approach}

$\mathrm{NN}$ are simple, but powerful and flexible tools for forecasting, provided that there are enough data for training, an adequate selection of the input-output samples, an appropriated number of hidden units and enough computational resources available.

Just like NN, a fuzzy logic system is a nonlinear mapping of an input vector into a scalar output, but it can handle numerical values and linguistic knowledge. 
A combination of $\mathrm{NN}$ and fuzzy systems has the advantages of each of them. In a neuro-fuzzy system, neural networks extract automatically fuzzy rules from numerical data and, through the learning process, the membership functions are adaptively adjusted.

ANFIS is a class of adaptive multi-layer feedforward networks, applied to nonlinear forecasting where past samples are used to forecast the sample ahead. ANFIS incorporates the self-learning ability of $\mathrm{NN}$ with the linguistic expression function of fuzzy inference [9].

The ANFIS architecture is shown in Figure 1. The ANFIS network is composed of five layers. Each layer contains several nodes described by the node function. The node function is described next. Let $O_{i}^{j}$ denote the output of the $i$ th node in layer $j$.

Layer 1 Layer 2 Layer 3 Layer 4 Layer 5

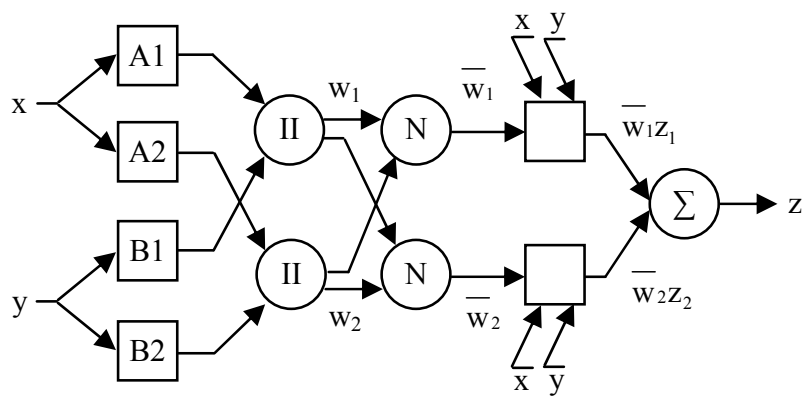

Fig. 1. ANFIS architecture.

In layer 1 , every node $i$ is an adaptive node with node function:

$O_{i}^{1}=\mu A_{i}(x), \quad i=1,2$

or

$O_{i}^{1}=\mu B_{i-2}(y), \quad i=3,4$

where $x$ (or $y$ ) is the input to the $i$ th node and $A_{i}$ (or $\left.B_{i-2}\right)$ is a linguistic label associated with this node.

Thus, $O_{i}^{1}$ is the membership grade of a fuzzy set $A$ (= $A_{1}, A_{2}, B_{1}$, or $B_{2}$ ) and it specifies the degree to which the given input $x$ (or $y$ ) satisfies the quantifier $A$. The membership functions for $A$ and $B$ are usually described by generalized bell functions, e.g.:

$$
\mu A_{i}(x)=\frac{1}{1+\left|\frac{x-r_{i}}{p_{i}}\right|^{2 q_{i}}}
$$

where $\left\{p_{i}, q_{i}, r_{i}\right\}$ is the parameter set. As the values of these parameters change, the bell-shaped function varies accordingly, thus exhibiting various forms of membership functions on linguistic label $A_{i}$.

In fact, any continuous and piecewise differentiable functions, such as triangular-shaped membership functions, are also qualified candidates for node functions in this layer [10]. Parameters in this layer are referred to as premise parameters.
In layer 2, each node $\Pi$ multiplies incoming signals and sends the product out:

$O_{i}^{2}=w_{i}=\mu A_{i}(x) \mu B_{i}(y), \quad i=1,2$

Hence, each node output represents the firing strength of a rule.

In layer 3, each node $\mathrm{N}$ computes the ratio of the $i$ th rules's firing strength to the sum of all rules' firing strengths:

$O_{i}^{3}=\bar{w}_{i}=\frac{w_{i}}{w_{1}+w_{2}}, \quad i=1,2$

The outputs of this layer are called normalized firing strengths.

In layer 4, each node computes the contribution of the ith rule to the overall output:

$$
O_{i}^{4}=\bar{w}_{i} z_{i}=\bar{w}_{i}\left(a_{i} x+b_{i} y+c_{i}\right), \quad i=1,2
$$

where $\bar{w}_{i}$ is the output of layer 3 and $\left\{a_{i}, b_{i}, c_{i}\right\}$ is the parameter set. Parameters of this layer are referred to as consequent parameters.

In layer 5 , the single node $\sum$ computes the final output as the summation of all incoming signals:

$$
O_{i}^{5}=\sum_{i} \bar{w}_{i} z_{i}=\frac{\sum_{i} w_{i} z_{i}}{\sum_{i} w_{i}}
$$

Thus, an adaptive network is functionally equivalent to a Sugeno-type fuzzy inference system.

The ANFIS considered in this study uses a hybrid learning algorithm to identify parameters of Sugeno-type fuzzy inference systems. Thus, ANFIS uses a combination of the least-squares method (to determine consequent parameters) and the backpropagation gradient descent method (to learn the premise parameters).

\section{Forecasting Accuracy Evaluation}

To evaluate the accuracy of the proposed neuro-fuzzy (NF) approach in forecasting wind power, different criterions are used. This accuracy is computed in function of the actual wind power that occurred. The mean absolute percentage error (MAPE) criterion, the sum squared error (SSE) criterion, and the standard deviation of error (SDE) criterion, are defined as follows.

The MAPE criterion is defined as follows:

$$
\begin{aligned}
\text { MAPE } & =\frac{100}{N} \sum_{h=1}^{N} \frac{\left|\hat{p}_{h}-p_{h}\right|}{\bar{p}} \\
\bar{p} & =\frac{1}{N} \sum_{h=1}^{N} p_{h}
\end{aligned}
$$

where $\hat{p}_{h}$ and $p_{h}$ are respectively the forecasted and actual wind power at hour $h, \bar{p}$ is the average wind power of the forecasting period and $N$ is the number of forecasted hours. 
The SSE criterion is given by:

$$
S S E=\sum_{h=1}^{N}\left(\hat{p}_{h}-p_{h}\right)^{2}
$$

The SDE criterion is given by:

$$
\begin{gathered}
S D E=\sqrt{\frac{1}{N} \sum_{h=1}^{N}\left(e_{h}-\bar{e}\right)^{2}} \\
e_{h}=\hat{p}_{h}-p_{h} \\
\bar{e}=\frac{1}{N} \sum_{h=1}^{N} e_{h}
\end{gathered}
$$

where $e_{h}$ is the forecast error at hour $h$ and $\bar{e}$ is the average error of the forecasting period.

\section{Numerical Results}

The proposed NF approach has been applied for wind power prediction in Portugal. Historical wind power data are the main inputs for training. For the sake of clear comparison, no exogenous variables are considered.

The forecast horizon is one day with a time-step of fifteen minutes. The following days are randomly selected: July 3, 2007, October 31, 2007, January 14, 2008, and April 2, 2008, corresponding to the four seasons of the year. Hence, days with particularly good wind power behavior are deliberately not chosen. This results in an uneven accuracy distribution throughout the year that reflects reality.

Numerical results with the proposed NF approach are shown in Figures 2 to 5 respectively for the winter, spring, summer and fall days. Each figure shows the actual wind power, solid line, together with the forecasted wind power, dash-dot line.

Table I presents the values for the criterions to evaluate the accuracy of the proposed NF approach.

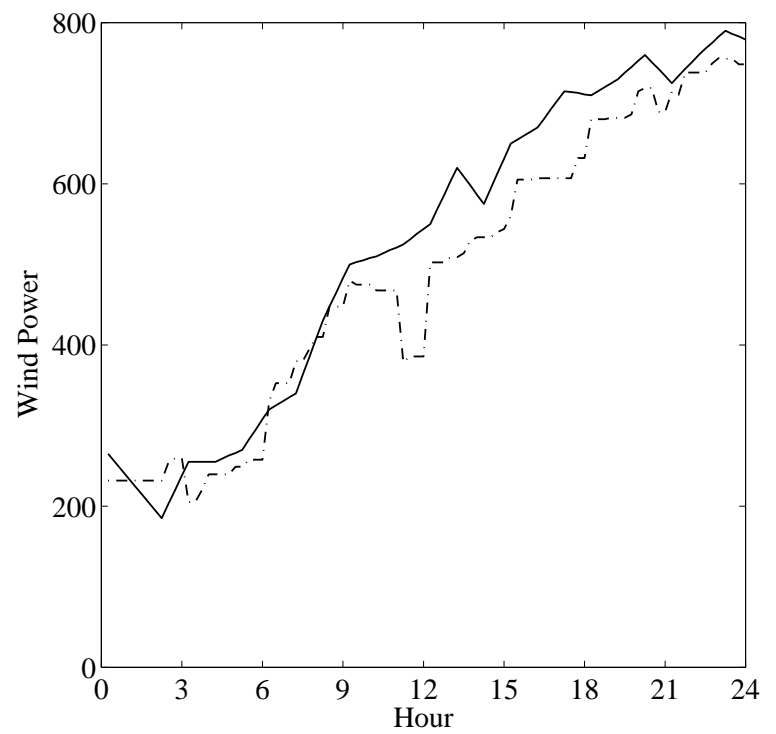

Fig. 2. Winter day: actual wind power with the forecasted wind power, in Megawatt.

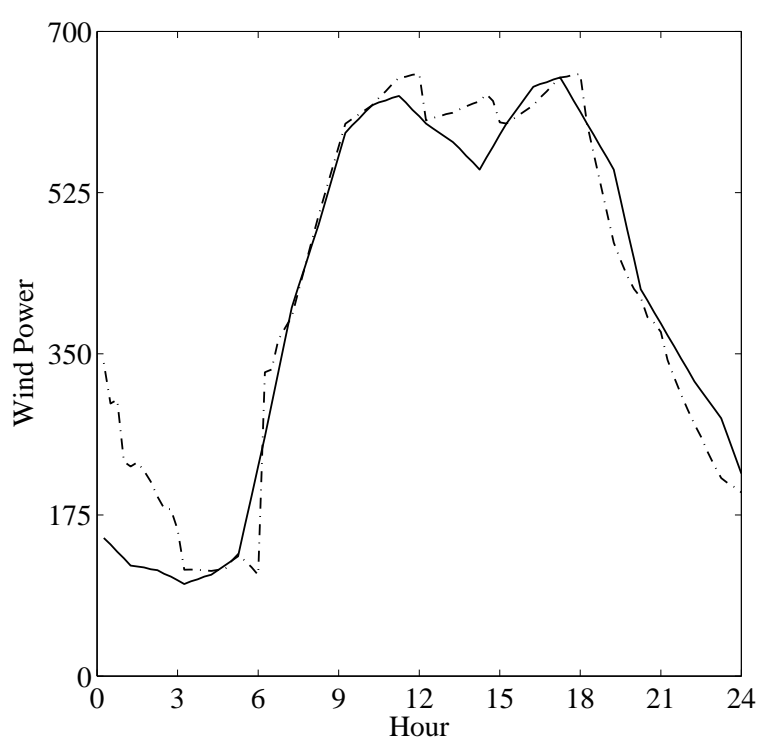

Fig. 3. Spring day: actual wind power with the forecasted wind power, in Megawatt.

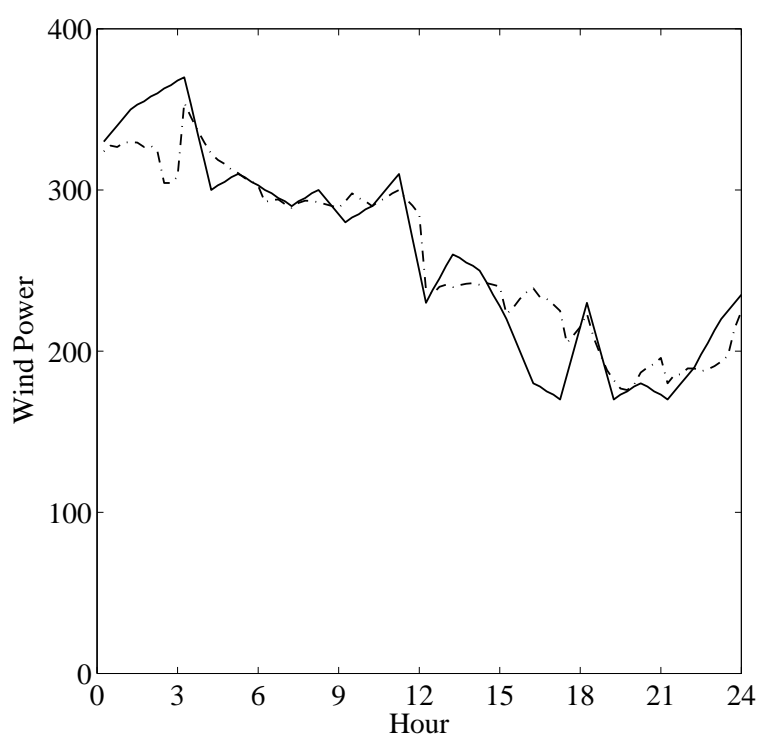

Fig. 4. Summer day: actual wind power with the forecasted wind power, in Megawatt.

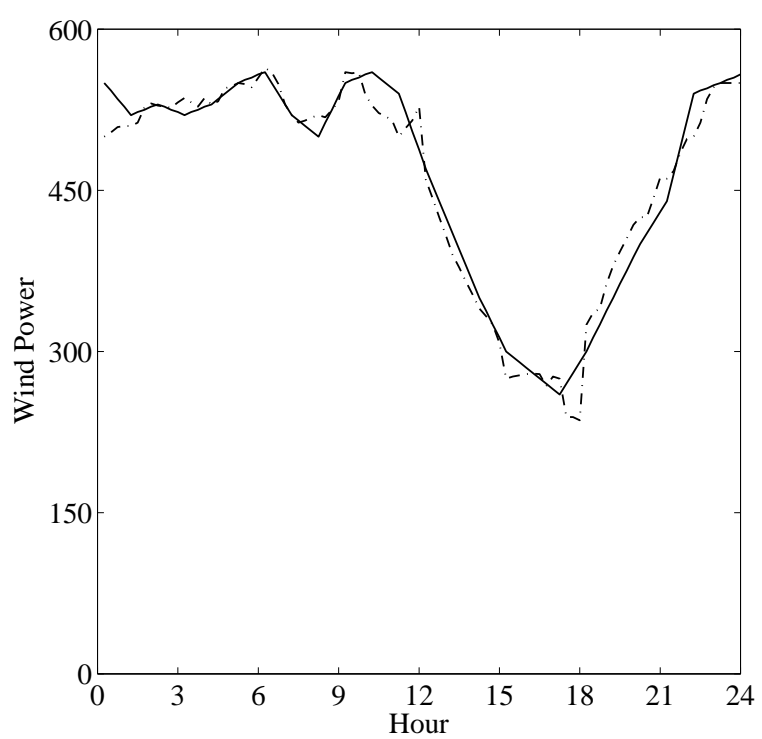

Fig. 5. Fall day: actual wind power with the forecasted wind power, in Megawatt. 
Table I. - Statistical Analysis of the Daily Forecasting Error

\begin{tabular}{|c|c|c|c|}
\hline Day & MAPE & $\sqrt{\mathrm{SSE}}$ & SDE \\
\hline Winter & 8.85 & 558.93 & 33.47 \\
\hline Spring & 8.96 & 520.42 & 38.15 \\
\hline Summer & 5.63 & 211.73 & 15.93 \\
\hline Fall & 3.11 & 187.61 & 12.82 \\
\hline
\end{tabular}

The proposed NF approach presents enhanced forecasting accuracy. The MAPE has an average value of $6.64 \%$. For comparison purposes, the average MAPE values for persistence, ARIMA and NN approaches would be $19.05 \%, 10.34 \%$ and $7.26 \%$, respectively. Moreover, the average computation time is less than 5 seconds, using MATLAB on a PC with $1 \mathrm{~GB}$ of RAM and a $2.0-\mathrm{GHz}-$ based processor.

\section{Conclusion}

As the penetration level of wind power in power systems increases, the accurate prediction of the wind behavior and the corresponding electric energy production will be increasingly important. In this paper, a NF approach is proposed for short-term wind power forecasting. The proposed NF approach outperforms persistence, ARIMA and NN approaches, regarding forecasting accuracy. Moreover, the average computation time is less than 5 seconds. The results confirm the considerable value of the proposed approach in forecasting wind power.

\section{References}

[1] T. Ackermann, "Wind Power in Power Systems", John Wiley and Sons Ltd.: West Sussex, 2005.

[2] A. Estanqueiro et al., "How to prepare a power system for $15 \%$ wind energy penetration: the Portuguese case study", Wind Energy, Vol. 11, pp 75-84, 2008.

[3] A. Costa et al., "A review on the young history of the wind power short-term prediction", Renew. Sust. Energy Rev., Vol. 12, pp 1725-1744, 2008.

[4] M. Lange and U. Focken, "Physical Approach to ShortTerm Wind Power Prediction", Springer-Verlag: Berlin, Heidelberg, 2006.

[5] R.G. Kavasseri and K. Seetharaman, "Day-ahead wind speed forecasting using f-ARIMA models", Renew. Energy, Vol. 34, pp 1388-1393, 2009.

[6] I.J. Ramirez-Rosado et al., "Comparison of two new shortterm wind-power forecasting systems", Renew. Energy, Vol. 34, pp 1848-1854, 2009.

[7] G. Sideratos and N.D. Hatziargyriou, "An advanced statistical method for wind power forecasting", IEEE Trans. Power Syst., Vol. 22, pp 258-265, 2007.

[8] R. Jursa and K. Rohrig, "Short-term wind power forecasting using evolutionary algorithms for the automated specification of artificial intelligence models", Int. J. Forecast., Vol. 24, pp 694-709, 2008.

[9] Z. Yun et al., "RBF neural network and ANFIS-based short-term load forecasting approach in real-time price environment", IEEE Transactions on Power Syst., Vol. 23, pp 853-858, 2008.

[10] J.-S.R. Jang, "ANFIS: adaptive-network-based fuzzy inference system", IEEE Transactions on Systems Man Cybernetics, Vol. 23, pp 665-685, 1993. 